\title{
BMJ Open Supporting breastfeeding In Local Communities (SILC) in Victoria, Australia: a cluster randomised controlled trial
}

\author{
Helen L McLachlan, ${ }^{1,2}$ Della A Forster, ${ }^{1,3}$ Lisa H Amir, ${ }^{1}$ Meabh Cullinane, ${ }^{1}$ \\ Touran Shafiei, ${ }^{1}$ Lyndsey F Watson, ${ }^{1}$ Lael Ridgway, ${ }^{2}$ Rhian L Cramer, ${ }^{1}$ \\ Rhonda Small ${ }^{1}$
}

To cite: McLachlan $\mathrm{HL}$, Forster DA, Amir LH, et al. Supporting breastfeeding In Local Communities (SILC) in Victoria, Australia: a cluster randomised controlled trial. BMJ Open 2016;6:e008292. doi:10.1136/bmjopen-2015008292

- Prepublication history and additional material is available. To view please visit the journal (http://dx.doi.org/ 10.1136/bmjopen-2015008292).

Received 24 March 2015 Revised 17 November 2015 Accepted 18 November 2015

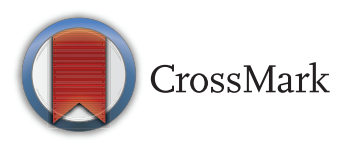

\footnotetext{
${ }^{1}$ Judith Lumley Centre, La Trobe University, Melbourne Victoria, Australia

${ }^{2}$ School of Nursing and Midwifery, Bundoora,

Victoria, Australia

${ }^{3}$ The Royal Women's

Hospital, Parkville, Victoria, Australia
}

Correspondence to Dr Helen L McLachlan; h.mclachlan@latrobe.edu.au

\section{ABSTRACT}

Objectives: Breastfeeding has significant health benefits for mothers and infants. Despite recommendations from the WHO, by 6 months of age $40 \%$ of Australian infants are receiving no breast milk. Increased early postpartum breastfeeding support may improve breastfeeding maintenance. 2 community-based interventions to increase breastfeeding duration in local government areas (LGAs) in Victoria, Australia, were implemented and evaluated.

Design: 3-arm cluster randomised trial.

Setting: LGAs in Victoria, Australia.

Participants: LGAs across Victoria with breastfeeding initiation rates below the state average and $>450$ births/ year were eligible for inclusion. The LGA was the unit of randomisation, and maternal and child health centres in the LGAs comprised the clusters.

Interventions: Early home-based breastfeeding support by a maternal and child health nurse (home visit, $\mathrm{HV}$ ) with or without access to a community-based breastfeeding drop-in centre (HV+drop-in).

Main outcome measures: The proportion of infants receiving 'any' breast milk at 3, 4 and 6 months (women's self-report).

Findings: $4 \mathrm{LGAs}$ were randomised to the comparison arm and provided usual care $(n=41$ clusters; $n=2414$ women); 3 to HV ( $n=32$ clusters; $n=2281$ women); and 3 to HV+drop-in ( $n=26$ clusters; 2344 women). There was no difference in breastfeeding at 4 months in either HV (adjusted OR 1.04; $95 \% \mathrm{Cl} 0.84$ to 1.29 ) or HV+drop-in (adjusted OR 0.92; 95\% Cl 0.78 to 1.08) compared with the comparison arm, no difference at 3 or 6 months, nor in any LGA in breastfeeding before and after the intervention. Some issues were experienced with intervention protocol fidelity.

Conclusions: Early home-based and community-based support proved difficult to implement. Interventions to increase breastfeeding in complex community settings require sufficient time and partnership building for successful implementation. We cannot conclude that additional community-based support is ineffective in improving breastfeeding maintenance given the level of adherence to the planned protocol.

\section{Strengths and limitations of this study}

- Supporting breastfeeding In Local Communities (SILC) was an adequately powered trial embedded within a universal state-wide maternal and child health $(\mathrm{MCH})$ service, and focused on the early postpartum period, when many women struggle most with breastfeeding.

- The interventions were delivered by specifically funded MCH nurses (SILC-MCHNs) with the skills and education required to support women to breast feed and whose role was to focus solely on breastfeeding.

- Substantial attention was given to process evaluation measures enabling thorough assessment of intervention fidelity.

- The local government areas involved were able to successfully add a new question assessing infant feeding 'in the past $24 \mathrm{~h}$ ' to the 4-month 'Key Ages and Stages' visit which gave better quality cross-sectional data, enhancing the capacity to discern changes due to the intervention. Use of unit-level routinely collected MCH data for SILC meant no additional data collection for participating MCHNs and missing data were minimal.

- SILC was a complex community-based randomised controlled trial and there were some unanticipated difficulties with intervention implementation: some women were not assessed for a SILC-MCHN visit and some visits did not occur as planned, soon after hospital discharge. Thus, the interventions were diluted, influencing both the reach and dose of the intervention.

Trial registration number: ACTRN12611000898954; Results.

\section{INTRODUCTION}

Breastfeeding is the number 1 preventive intervention in child health. ${ }^{1}$ However, despite recommendations from the WHO and national authorities, ${ }^{2}{ }^{3}$ United Nations 
Children's Fund (UNICEF) reports little change in exclusive breastfeeding in the past 20 years. ${ }^{1}$ Only $16 \%$ of Australian infants receive just breast milk until 6 months. ${ }^{4}$ Even more striking is the increasing gap in breastfeeding rates between the most and least disadvantaged families. ${ }^{5}$ In the state of Victoria in Australia, breastfeeding rates show marked disparities in the proportion of infants receiving any breast milk at 6 months of age in different local government areas (LGAs), ranging from $32 \%$ to $68 \%{ }^{6}$

A large body of evidence describes interventions which promote the initiation and/or duration of breastfeeding, including several Cochrane reviews. ${ }^{7-11}$ Current evidence suggests that effective strategies for increasing breastfeeding duration include face-to-face and proactive support. ${ }^{10}$ In Australia, no community-based trials of potentially sustainable, community-level interventions were identified.

In Victoria, universal community-based, governmentfunded support is provided by the maternal and child health $(\mathrm{MCH})$ service for families with children from birth to school age, so provides a suitable platform for the conduct of a community-based early intervention to support breastfeeding. ${ }^{12} \mathrm{MCH}$ centres are located throughout local communities to enhance accessibility. Ten consultations with MCH nurses (MCHNs; registered nurses with additional midwifery and child and family health qualifications) are offered to parents, known as Key Ages and Stages (KAS) visits, beginning with a home visit (HV) at 1-2 weeks postpartum, followed by five centre-based visits over the first 8 months.

The Supporting breastfeeding In Local Communities (SILC) trial was designed to investigate whether early breastfeeding support at home, for women most at risk of breastfeeding cessation, with and without access to breastfeeding assistance in drop-in centres, would increase breastfeeding maintenance in Victorian LGAs with low breastfeeding rates. ${ }^{13}$

\section{METHODS}

A three-arm cluster randomised controlled trial (RCT) design was used: a comparison (usual care) arm and two intervention arms. The first intervention was proactive, early MCHN home-based breastfeeding support (HV). The second included early home-based support, with an additional component-access to a community-based breastfeeding drop-in centre (HV+drop-in). The three-arm design enabled us to draw conclusions about the effect of either a single strategy, or the implementation of two approaches at once in relation to standard care.

\section{Study hypotheses}

\section{Primary hypotheses}

Intervention LGAs providing $\mathrm{HV}$ and $\mathrm{HV}+$ drop-in will have a higher proportion of infants receiving any breast milk at 4 months compared with LGAs providing usual $\mathrm{MCH}$ care.

\section{Secondary hypotheses}

Intervention LGAs providing $\mathrm{HV}$ and $\mathrm{HV}+$ drop-in will have a higher proportion of infants receiving any breast milk at 3 and 6 months compared with LGAs providing usual MCH care.

\section{Study population}

All eligible LGAs in Victoria, Australia, were invited to participate. Eligibility criteria were having a lower rate of any breastfeeding at discharge from hospital than the Victorian state average; and more than 450 births per year. ${ }^{14}$ LGAs were excluded if they had breastfeeding initiatives in place similar to the proposed interventions.

\section{Intervention allocation}

Eligible LGAs were randomly allocated to one of the three trial arms, stratified by the number of births per year (large $>2500$; medium 1000 to 2500 ; small $<1000$; figure 1). Allocation to trial arms took place using opaque envelopes at a state-wide MCH forum. Individual randomisation was not considered practical in the study context. Further detail is provided in the study protocol. $^{13}$

\section{Usual care}

Services in LGAs allocated to 'usual care' were those routinely available to women after birth in Victoria. These included a hospital midwife visit/s 1-2 days after discharge, with the usual length of stay in hospital after the birth being $48 \mathrm{~h}$ or less, and with a general focus on the well-being of mother and infant. These visits are for women who have public maternity care; this service is generally not provided for women accessing private maternity care, which is approximately one-third of women in Victoria. ${ }^{15}$ Women also routinely receive an MCHN home visit, usually 10 days to 2 weeks after birth, with breastfeeding assessment, support and advice a core component of care. Thus, there is often a gap of 510 days between the hospital midwife visit and the time of the MCHN home visit. Women access MCH centrebased care thereafter. Other community supports include a state-wide $24 \mathrm{~h}$ MCH helpline; a $24 \mathrm{~h}$ Australian Breastfeeding Association (ABA) helpline; and support by general practitioners and other health professionals as sought by families. Women receiving care in participating LGAs would have given birth in a wide variety of hospitals; it is therefore not possible to describe the types of breastfeeding supports that may have been offered by different hospitals, nor whether women had public or private care.

The system by which LGAs are notified of women's births is similar across the state. The hospital where the woman gives birth is required to notify the appropriate LGA of the birth, to trigger the LGA to start the universal care. The system is usually electronic, with an expectation that there would be a more one-to-one hand over (such as a telephone conversation) if that was required, for example, for a woman with complex needs. LGAs 


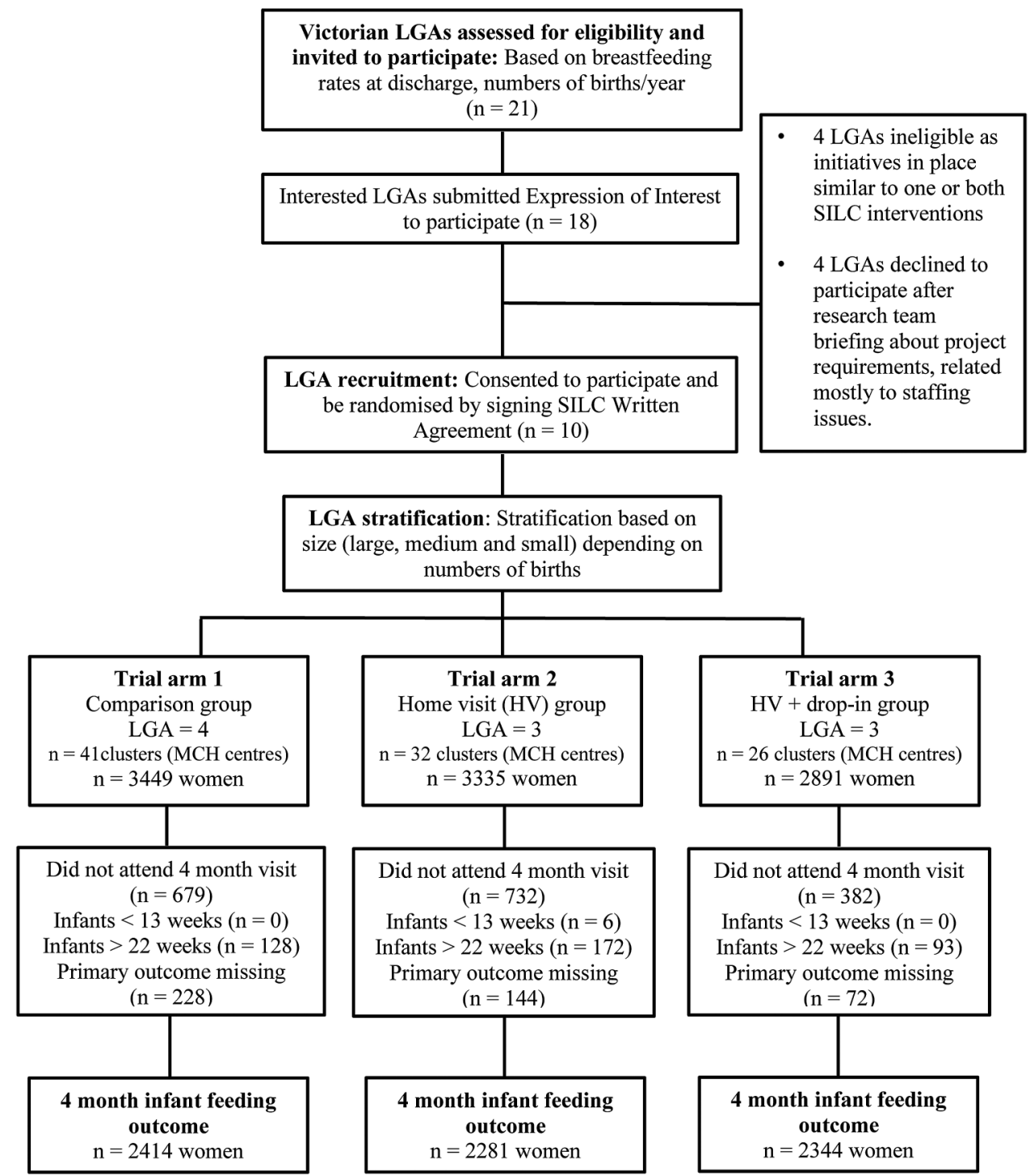

Figure 1 CONSORT flow chart (LGA, local government area; $\mathrm{MCH}$, maternal and child health; SILC, Supporting breastfeeding In Local Communities.

each have systems in place to ensure that women's information is communicated to the appropriate $\mathrm{MCH}$ local centre and relevant MCHN. The usual process after this is that all women are telephoned and an appointment time made for the MCHN to visit women at home by 10-14 days after birth. In some LGAs, these calls are made by $\mathrm{MCH}$ local centre nurses, and in others the process is centralised.

\section{Interventions}

Intervention LGAs provided all usual MCH care, as described above with the addition of $\mathrm{HV}$ or $\mathrm{HV}+$ drop-in.

\section{HV (early home-visiting only)}

LGAs allocated to the HV trial arm provided early home-based visiting by a MCHN to women identified at risk of breastfeeding cessation. MCHNs were specifically employed from within the LGAs; prior to the start of intervention they attended $6 \mathrm{~h}$ of workshops/ training regarding intervention implementation (SILC-MCHNs). ${ }^{13}$ The SILC-MCHN HV was arranged during the MCH service's first contact with the woman after hospital discharge, aimed at providing proactive breastfeeding assistance as early as possible after birth. The aim was to fill the gap that currently exists between cessation of hospital-based care and start of MCH care (usually 7 days or more). LGAs were asked to undertake the 'routine' telephone call as early as possible postpartum in order to assign women an early SILC-MCHN visit if required, and much of the education focus of the trial was on this aspect.

The focus of the SILC-MCHN HVs was the normalisation of breastfeeding, building women's confidence to breast feed, reassurance, development of an infant feeding plan (where needed), and provision of a list of useful websites and telephone numbers. The topics covered at individual visits were driven by the specific needs of the woman, with no set way of approaching 
issues. Women were referred to additional services as needed. SILC-MCHNs were MCHNs with additional training in, and a commitment to, supporting breastfeeding. All were required to attend a 1-day workshop prior to the start of the interventions to introduce them to the study and to RCTs generally, as well as to provide guidance on how to provide and document the intervention, and the importance of a woman-centred approach. They also attended three further half days of education, feedback and support throughout the intervention phase.

\section{$H V+d r o p-i n$ (early home-visiting plus access to a breastfeeding drop-in centre)}

In addition to SILC-MCHN early home-based breastfeeding support, LGAs allocated to the HV+drop-in trial arm established a local community breastfeeding drop-in centre staffed by a SILC-MCHN, and where possible with a trained peer supporter or community educator or counsellor.

The drop-in centres were welcoming spaces offering privacy, where women could discuss breastfeeding concerns with the SILC-MCHN, with access to drinks, change tables and toilets, and the opportunity to meet and learn from other mothers. Women were informed about drop-in centres in a range of ways. This included written information provided to women at the hospital where they gave birth, distribution of fliers to new mothers by $\mathrm{MCH}$ nurses, and displaying of posters in $\mathrm{MCH}$ centres, medical clinics, kindergartens and childcare centres. There was also some advertising in local print media and some broadcasts on local radio. LGAs were given autonomy in terms of where the drop-in centres were located and how women were informed about them. Education and discussion of these processes was undertaken at all trial stages-during the call for expressions of interest, in the briefings regarding what trial participation would involve, and extensively during the run-in period and early stages of the interventions. LGAs were allocated funds to cover staffing of the drop-in centres based on their birth numbers, and were able to make their own choices as to the centre hours. The infrastructure cost of the drop-in centres had to be covered at the LGA level. We anticipated that on average drop-in centres would be able to run for two half days per week in small and medium LGAs and three half days in the large LGAs.

\section{Determining eligibility for SILC-MCHN HV}

It was planned that within each intervention LGA, SILC-MCHNs would conduct an average of two home visits to approximately $30 \%$ of women who left hospital breastfeeding. This proportion was chosen pragmatically, both to identify those at risk of early cessation and/or experiencing problems leading to early cessation and to enhance sustainability (ie, by visiting only those at highest risk of early cessation, to conserve resources) if the intervention proved effective. There is strong evidence that infants receiving formula in the early postpartum period are at higher risk of premature cessation of breastfeeding than other infants, ${ }^{16} 17$ and approximately $14 \%$ of Victorian-born infants are being supplemented with infant formula in the early days postpartum. ${ }^{14}$ It is also known that around $30 \%$ of women self-identify breastfeeding problems in the first few days postpartum, ${ }^{18}$ though on its own this is unlikely to be a reliable indicator for risk of early cessation. Therefore, we aimed to identify and conduct visits to the $30 \%$ of women who are most at risk of early breastfeeding cessation, using a mix of eligibility criteria.

An assessment tool was developed and tested in nontrial LGAs to determine eligibility for a SILC-MCHN visit. The aim was to provide proactive support to the women most likely to cease breastfeeding. A visit would be arranged:

- If a woman's infant received any infant formula in addition to breast milk (either at the breast or expressed breast milk (EBM)), in the $24 \mathrm{~h}$ prior to telephone contact; or

- If a woman was distressed about breastfeeding or asked for help with breastfeeding when telephoned, even if she was not supplementing with infant formula.

MCHNs could also identify women for a SILC-MCHN home visit at a later standard $\mathrm{MCH}$ visit, if needed. In LGAs where infant formula use was consistently ascertained as less than $25 \%$ of women who left hospital breastfeeding, EBM use in the $24 \mathrm{~h}$ prior to telephone contact (whether or not infant formula had been given) was included in the assessment criteria, as this is also known to be a risk factor for early breastfeeding cessation. $^{19}$

We did extensive onsite education with the MCHNs in all LGAs, in addition to the specific SILC-MCHN education. We aimed to ensure that all those involved would have a clear understanding of the eligibility assessment process, the referral pathways and the intervention.

\section{Sample size}

This study aimed to detect a difference in any breastfeeding from $48 \%$ (the estimated rate of any breastfeeding at 4 months in those LGAs below the state average breastfeeding rate at hospital discharge) to $58 \%$ at 4 months. As the tested intervention would only have the ability to affect women who were breastfeeding at discharge (ie, not women who chose to formula feed; and in participating LGAs, the average rate of any breastfeeding at hospital discharge was $81 \%$ ), to gain a $10 \%$ increase, the difference in the rate of breastfeeding was estimated at $59 \%$ (or $48 \% / 81 \% \times 100)$ to $72 \%(58 \% / 81 \% \times 100)$.

For a simple random sample, this required 224 women in each intervention arm (with $\alpha 0.05$ and $80 \%$ power), that is, 448 in total. The LGAs were the unit of randomisation, and the multiple $\mathrm{MCH}$ centres within formed the clusters for analysis. To take into account the potential effect of clustering, we inflated the sample size 
assuming an intracluster correlation (ICC; r) of 0.02 , thus requiring 400-500 women in each intervention arm. Up to four LGAs per trial arm were needed to achieve this. See the study protocol for more detail on sample size calculations and assumptions. ${ }^{13}$

\section{Data collection}

The interventions ran in the LGAs for a 9-month period from July 2012 to March 2013. The first 2 months formed a pilot phase and a run-in period to allow time for the LGAs to establish the interventions.

\section{Primary outcome (measured at the individual, not cluster, level)}

The primary outcome of the study was any breastfeeding at 4 months. We chose 4 months as the primary outcome point because breastfeeding at 4 months is a key national indicator of children's health, development and wellbeing, and thus this is the time the visit is scheduled to occur (noted in trial protocol paper). The feeding data collected for 6 months in the LGA context are actually asked at the 8 month visit. Given we wanted to ask about infant feeding in the past $24 \mathrm{~h}$, we therefore chose 4 months. The participation rate is also far higher at that visit $(>93 \%)$ compared with the later visit $(\sim 85 \%)$.

A single data item asking about infant feeding in the past $24 \mathrm{~h}$ ' was added to the usual questions asked by
MCHNs, to ascertain this outcome at the routine 4-month KAS visit (figure 2).

\section{Baseline and outcome infant feeding data collection}

Baseline breastfeeding outcomes (3, 4 and 6 months) were collected in all participating LGAs for a period of 3 months before infants exposed to the interventions had their 4-month MCH appointments. The same new single data item (described above) asking about infant feeding 'in the past $24 \mathrm{~h}$ ' was used for the 4-month baseline measure, and routinely collected infant feeding data were used for the 3-month and 6-month baseline and outcome measures. These data were used to assess comparability of trial arms at the start of the trial and to enable a before and after analysis of breastfeeding rates at 3, 4 and 6 months in each LGA. Outcome infant feeding data collection began directly after this period.

\section{Other variables of interest}

A range of other variables of interest (or potential confounders (known to affect breastfeeding) for the primary outcome) were abstracted from routinely collected MCH data. ${ }^{13}$ These included gestational age and date of birth of the infant; mode of birth; maternal parity, age, country of birth, and if born overseas, year of arrival; country of birth of father; $\mathrm{MCH}$ centre postcode; $\mathrm{MCH}$ centre code (the cluster variable); date of routine

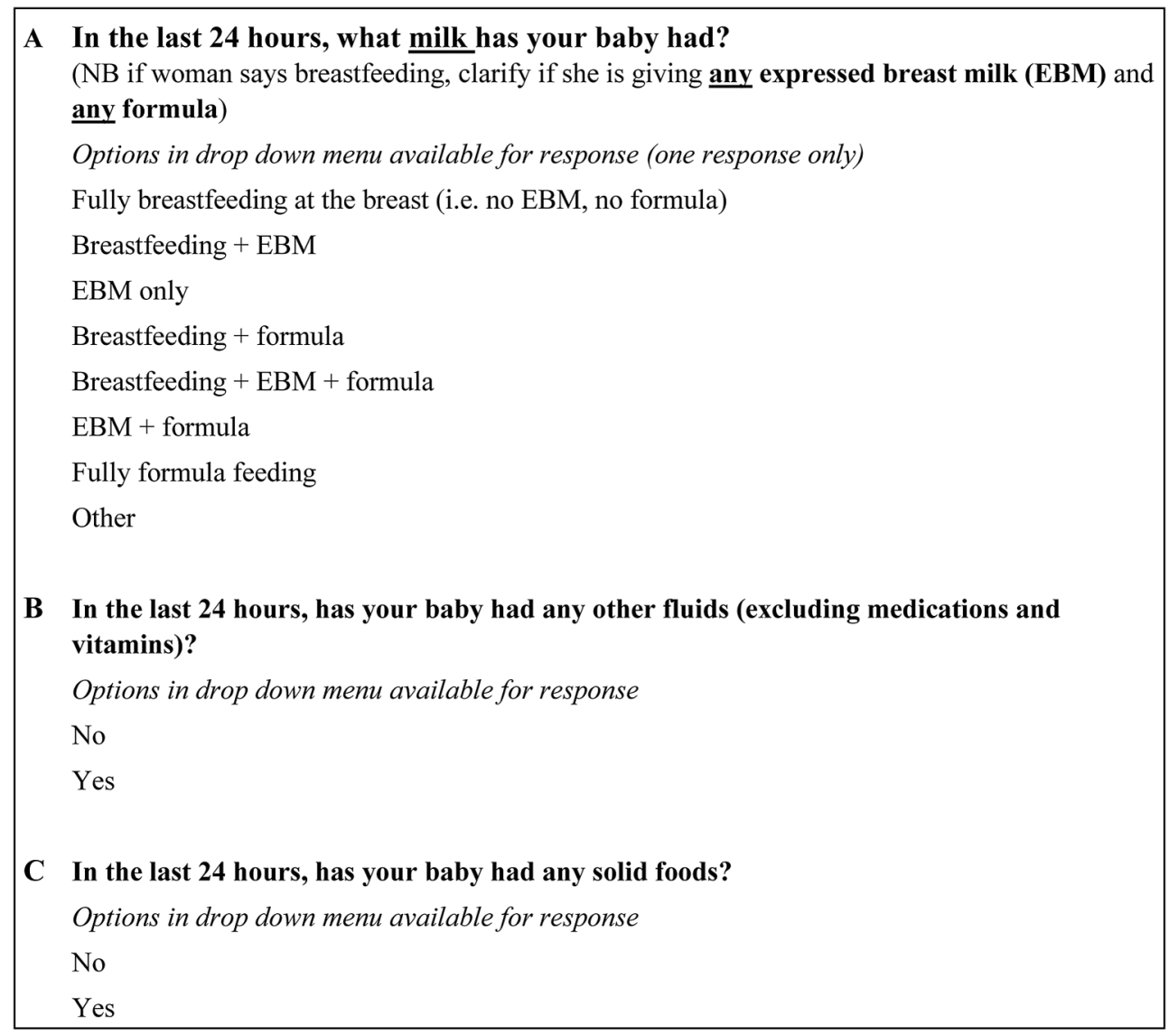

Figure 2 Primary outcome data collection question, asked at routine 4-month maternal and child health visit. 
4-month KAS visit; infant age in weeks at the routine 4-month KAS visit; Health Care Card status (concession card for low-income earners); and Aboriginal or Torres Strait Islander status of mother.

\section{Assessment of intervention fidelity}

Intervention implementation documentation included number of women assessed as eligible for a SILC-MCHN visit; number of women visited; attendance at drop-in centres; and details of the interventions provided. ${ }^{13}$

\section{Data analysis}

Stata 11 was used for all analyses. Each intervention group was compared with the comparison care group using intention-to-treat analysis, with analyses conducted blinded to group allocation.

\section{Primary outcome}

Proportions of women giving their baby any breast milk at 4 months were compared using logistic regression and both ORs and adjusted ORs (adj ORs) are presented. Women with infants whose age was $<13$ and $>22$ weeks were excluded from the analysis (given their inclusion might result in overestimating or underestimating the percentage giving any breast milk at 4 months). For baseline differences in potential confounders between intervention and comparison groups that might be associated with breastfeeding outcomes, an online supplementary multivariate analysis was carried out. In all analyses, adjustment was made for clustering at the $\mathrm{MCH}$ centre level as well as baseline breastfeeding rates. This was done using the 'survey set' command in Stata prior to all regression. MCH centre codes were used to denote clusters, and the three LGA sizes (large, medium and small, based on birth numbers) were assigned as strata.

ICCs were calculated using post-intervention (primary outcome) data, with no adjustment for covariates, and are reported along with the primary outcomes. Given our primary outcome variable was binary, we used a multilevel logistic regression model, which was implemented in
Stata using the 'melogit' command, followed by 'estat icc'. Point estimates as well as 95\% CIs are reported, along with average cluster size and range. ${ }^{20}$

\section{Secondary outcomes}

Proportions of women giving their baby any breast milk at 3 and 6 months were compared using logistic regression and ORs presented.

A secondary analysis was conducted to explore factors independently associated with being less likely to be breastfeeding at 4 months. Available explanatory variables were tested at the univariate level, and those associated with any breastfeeding were included in a single model. Variables were removed in a stepwise manner until only those that were significant at the 0.05 level were retained.

\section{Ethical considerations}

Approval for the study was granted by the La Trobe University Human Ethics Committee (project number 11-068) and the Department of Education and Early Childhood Development (project number 2011_ 001305). Individual consent was not required-LGAs provided written consent, and in those LGAs that were allocated to the intervention, the intervention was considered 'standard' care.

\section{RESULTS}

Twenty-one of 79 Victorian LGAs were assessed as eligible and invited to participate, and 10 consented and were randomised. Figure 1 illustrates this process and shows the flow of participants through the trial. The number of clusters in each trial arm is shown, along with the number of new infants registered by the $\mathrm{MCH}$ centres in each trial arm. A small number of women in each trial arm were excluded from data analysis as their infant was $<13$ or $>22$ weeks of age at the time of the routine 4-month $\mathrm{MCH}$ visit, and the primary outcome measure was not reported in some records. The larger

Table 1 Demographic characteristics by trial arm

\begin{tabular}{|c|c|c|c|}
\hline Characteristic & $\begin{array}{l}\text { Comparison arm } \\
n(\%) n=2642\end{array}$ & $\begin{array}{l}\text { Home visit (HV) arm } \\
n(\%) n=2425\end{array}$ & $\begin{array}{l}\text { HV plus drop- } \\
\text { in centre arm } \\
n(\%) n=2416\end{array}$ \\
\hline Maternal age (years), mean (SD) & $30.7(5.3)$ & $31.1(5.0)$ & $31.4(5.1)$ \\
\hline Infant age at $4 \mathrm{~m}$ KAS visit (weeks), mean (SD) & $17.7(1.2)$ & $17.8(1.3)$ & $17.8(1.1)$ \\
\hline Gestational age (weeks), mean (SD) & $39.1(1.6)$ & $39.1(1.6)$ & $39.0(1.5)$ \\
\hline Caesarean birth $(2565 / 2344 / 2320)^{*}$ & 826 (32.2) & $811(34.6)$ & $829(35.7)$ \\
\hline First time mother $(2642 / 2425 / 2416)^{\star}$ & 1037 (39.3) & 1001 (41.3) & $1017(42.1)$ \\
\hline Born in Australia $(2603 / 2425 / 1303)^{*}$ & $1795(69.0)$ & $1407(58.0)$ & $949(73.8)$ \\
\hline Health Care Card holder $(1923 / 1877 / 596)^{*}$ & 219 (11.4) & $342(18.2)$ & $97(16.3)$ \\
\hline $\begin{array}{l}\text { Aboriginal or Torres Strait Islander } \\
\text { mother }(2596 / 2425 / 1084)^{\star}\end{array}$ & $35(1.4)$ & $16(1.0)$ & $17(1.6)$ \\
\hline
\end{tabular}


difference between infants registered and those with a visit recorded at 4 months is likely to be due to nonattendance at the 4-month visit.

Demographic data were similar across the three trial arms (table 1) with the exception of the proportion of Australian-born mothers $(69 \%$ in comparison LGAs; $58 \%$ in home-visiting LGAs; $73 \%$ in home-visiting plus drop-in centre LGAs).

\section{Primary outcome: any breast milk feeding at 4 months postpartum}

When adjustments for baseline breastfeeding rates in the LGAs, gestational age, maternal age, caesarean births, age in weeks at the 4 month KAS visit and the potential effect of clustering in the $\mathrm{MCH}$ centres were taken into account, there was no difference in any breast milk feeding at 4 months between the comparison and home-visiting trial arms (adj OR 1.04, 95\% CI 0.84 to 1.29) or the comparison and home-visiting plus drop-in trial arms (adj OR 0.92, 95\% CI 0.78 to 1.08; table 2). The other three demographic variables available (maternal country of birth, aboriginality and Health Care Card holder status) had large amounts of missing data, as can be seen in table 1 . These were therefore not included in the final adjusted analysis. Including the three variables in the analysis changed the CIs slightly, but the overall result was unchanged; the interventions made no difference compared with the comparison communities.

\section{Intracluster correlation}

There were 99 clusters in total. The average cluster size was 71.1 (range 1-244), and the ICC was 0.03 (95\% CI 0.02 to 0.05$)$. In the control arm, there were 41 clusters, with an average of 58.9 women per cluster (range 9213 ), and the ICC was 0.02 (95\% CI 0.005 to 0.05 ). The home-visiting arm had 32 clusters of 71.3 women on average (range 1-226), and an ICC of 0.03 (95\% CI 0.01 to 0.06). The home-visiting plus drop-in arm had 26 clusters, with an average of 90.2 women per cluster (range 2-244), and an ICC of 0.03 (95\% CI 0.01 to $0.07)$.

\section{Secondary outcomes}

A before and after analysis of any breast milk feeding at baseline in each trial arm and in each LGA compared with the same outcome at 3 and 6 months postpartum found no difference in any breastfeeding before and after the SILC interventions (table 3).

A before and after analysis of any breast milk feeding at 4 months postpartum in each trial arm and individual LGAs was also conducted (in addition to adjusting for baseline breastfeeding rates in the primary analysis). There were no significant differences between any breast milk feeding at baseline and after the SILC interventions in any trial arm or any individual LGA (figure 3).

\section{Factors associated with any breastfeeding at 4 months of age (secondary analysis)}

Given there was no difference in the primary outcome by trial arm, the data were pooled to explore factors independently associated with any breastfeeding at 4 months. Several maternal factors were independently associated with infants being significantly less likely to be receiving any breast milk at 4 months postpartum (table 4). These included being less than 25 years of age; a first time mother; Australian-born; having a Health Care Card and being of Aboriginal or Torres Strait Islander origin. Other (non-maternal) factors included giving birth at less than 37 weeks gestation, having a caesarean birth and an infant's father being Australian-born. When including these factors in a single model, three factors were no longer associated with not receiving breast milk at 4 months: being a first time mother, being an infant of an Aboriginal or Torres Strait Islander woman (due to very small numbers); and infants with Australian-born fathers (due to the high correlation of this factor with having an Australian-born mother). All others remained significantly associated with lower breastfeeding rate at 4 months postpartum.

\section{Implementation of the interventions \\ Assessment for SILC-MCHN HVs}

Table 5 shows the number of women in the intervention trial arms who were registered for care in the $\mathrm{MCH}$ centres, the number of these estimated to be

Table 2 Primary outcome: any breast milk feeding at 4 months of age

\begin{tabular}{|c|c|c|c|c|c|c|}
\hline \multirow[b]{2}{*}{ Trial arm } & \multicolumn{2}{|c|}{ Any breast milk feeding } & \multirow[b]{2}{*}{ OR $^{*}$} & \multirow[b]{2}{*}{ Adjusted OR† } & \multirow[b]{2}{*}{$95 \% \mathrm{Cl}$} & \multirow[b]{2}{*}{ p Value } \\
\hline & $\mathrm{n} \%$ & (baseline \% ‡) & & & & \\
\hline Comparison arm $(n=2414)$ & 1300 (53.9) & $(54.1)$ & Ref & Ref & & \\
\hline Home visit arm $(n=2281)$ & $1429(62.7)$ & (63.3) & 1.44 & 1.04 & 0.84 to 1.29 & 0.71 \\
\hline $\begin{array}{l}\text { Home visit plus drop-in centre } \\
\text { arm }(n=2344)\end{array}$ & $1276(54.4)$ & (57.2) & 1.02 & 0.92 & 0.78 to 1.08 & 0.30 \\
\hline \multicolumn{7}{|c|}{$\begin{array}{l}\text { *Unadjusted OR. } \\
\text { †Adjusted OR: adjusted for baseline breastfeeding rates in the local government areas, gestational age, maternal age, caesarean births, age } \\
\text { in weeks at } 4 \text { m Key Ages and Stages visit, and the potential effect of clustering in the maternal and child health centres (in regression } \\
n=4481 \text { in comparison }(n=2312) \text { to home visits }(n=2169) \text { trial arm, and } n=4471 \text { in comparison to }(n=2312) \text { home visits }+ \text { drop-in centre } \\
(n=2159) \text { trial arm-these numbers are less than shown in table due to variables with missing data being omitted in regression model). } \\
\ddagger \text { Đaseline-prior to start of interventions. }\end{array}$} \\
\hline
\end{tabular}




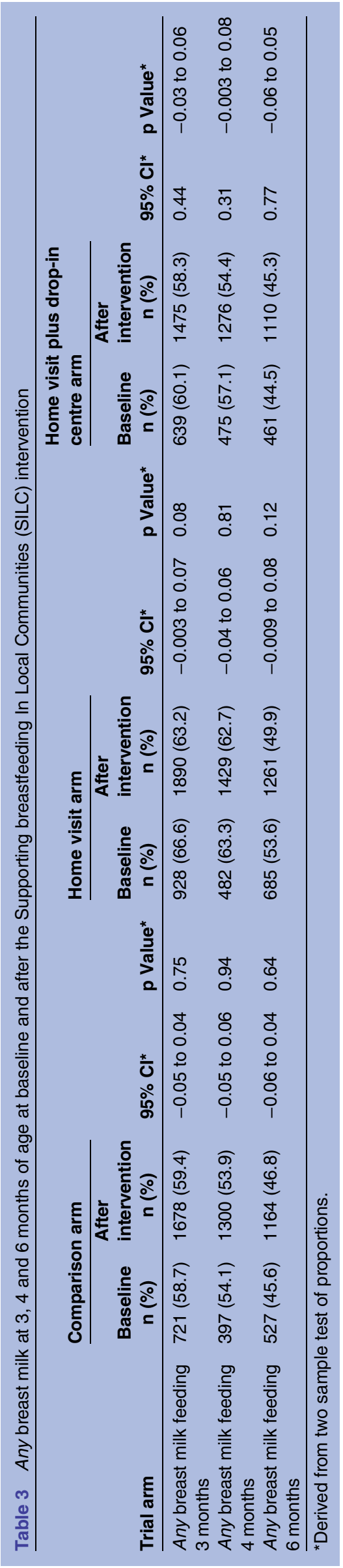

breastfeeding, and the subsequent number and percentage of these who were assessed for a visit (63\% overall) and had a visit arranged ( $16 \%$ overall). Considering the six intervention LGAs, assessment of women eligible for a SILC-MCHN visit ranged from $53 \%$ to $84 \%$ in the six LGAs (complete data by LGA not shown in paper, just trial arm data (table 5)). Between $16 \%$ and $33 \%$ of women declined a SILC-MCHN visit, whereas extremely few women declined the usual MCHN home visit

\section{SILC-MCHN HV implementation}

Overall, just $20 \%$ of women (1043/5317) who were breastfeeding at hospital discharge in the intervention LGAs received a SILC-MCHN home visit. Of these, 278 $(27 \%)$ received at least a second visit, that is, $5 \%$ of women breastfeeding at hospital discharge $(278 / 5317)$ received two or more home visits. Nineteen per cent of women $(193 / 1035)$ received a visit within the first week after giving birth.

\section{Drop-in centres}

In all three LGAs allocated to HV+drop-in, the SILC drop-in centres were poorly attended, with one attendance per session on average, and in two, peer supporters to staff the drop-in centres could not be recruited. Detailed implementation data on the drop-in centres will be reported separately.

\section{DISCUSSION}

This large cluster RCT found no difference in any breast milk feeding at 3, 4 or 6 months across trial arms. There were also no differences in the before and after breastfeeding rates in any trial arm or within LGAs.

SILC is the largest breastfeeding RCT conducted to date in Australia, and the first conducted in the community. Like most breastfeeding RCTs conducted in Australia, it had no impact on breastfeeding maintenance. The previous Australian RCTs were mostly inadequately powered; just two had a sufficient sample size to find a $10 \%$ difference in breastfeeding if one existed. ${ }^{21}{ }^{22}$ Only one found increased breastfeeding initiation, but this was in a specific cultural group. ${ }^{23}$ Another increased breastfeeding at 6 weeks, ${ }^{24}$ a result not replicated in another trial that retested the intervention. ${ }^{21}$ On the other hand, a Cochrane review of breastfeeding support for new mothers which included 52 trials from 21 countries (including 8 cluster trials and 37 trials from high-income countries) concluded that all types of extra support increased any breastfeeding, although the effect was modest. ${ }^{10}$ The quality of included studies was mixed, and most had methodological weaknesses. The review found that the greatest effects were in communities with high levels of breastfeeding initiation (over $80 \%$ ). ${ }^{10}$

Although our study was based on strategies that appeared to have some promise, including peer/lay and professional support, and used multistrategy interventions, there was little local Australian data to guide 
Figure 3 Any breast milk feeding at 4 months of age at baseline and post-intervention in all local government areas (LGAs). Baseline: $n=397$ in comparison arm; $n=482$ in home visit arm; $n=475$ in home visit plus drop-in centre arm. Intervention: $\mathrm{n}=1300$ in comparison arm; $n=1429$ in home visit arm; $n=1276$ in home visit plus drop-in centre arm.

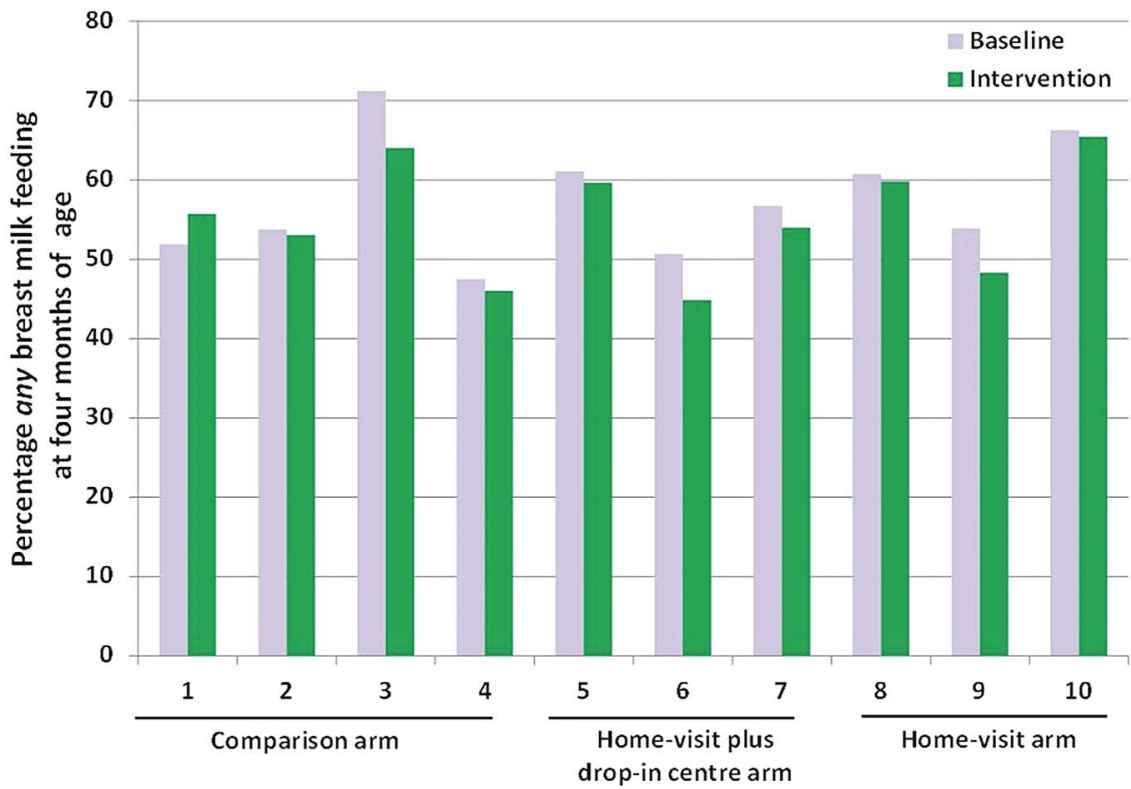

LGA potential strategies. We aimed to address the pivotal point where new mothers are no longer under hospital care and are at risk of ceasing breastfeeding, ${ }^{25}$ as well as providing support in a critical early period where there is a service gap.

SILC was a complex intervention introduced into a complex real-life community setting, with several 'active' ingredients. ${ }^{26}$ Our process evaluation data demonstrate that a range of factors affected intervention fidelity and thus impacted on the overall dose and reach of the interventions, despite all participating LGAs being very engaged in the intervention concept and processes. Most LGAs were unable to assess all women for eligibility for early breastfeeding support; they provided fewer home visits than planned, and most were later than planned. This was despite numerous visits by the study team to the trial sites, and extensive discussion and education. It is not clear why this was the case, and it is likely to be more than one factor, with influences such as staff workloads, not receiving information on births in a timely manner, and possibly a perception that women did not want or need support in addition to usual care. It may also be that LGAs were more likely to visit those families that were easiest to contact; in some cases only one attempt to telephone women was made, and if there was no contact then the opportunity to intervene early was lost. There were also challenges with drop-in centre establishment, including recruitment of volunteers, and poor attendance. At times staff (both administrative and SILC-MCHNs) were not replaced while on leave, which may have affected home visits and drop-in centre hours. These contextual factors appeared to have an impact on the implementation of SILC. This was also the case in a cluster trial of community-based breastfeeding support programmes in Scotland, which found a decline in breastfeeding rates in three of seven intervention localities. ${ }^{27}$ In that study, breastfeeding was negatively impacted by factors such as poor interprofessional communication, staff shortages, high workload and poor morale, and breastfeeding increased where there was more evidence of leadership, a focus on policy, multidisciplinary partnerships and reflective action cycles. ${ }^{27}$ Similarly, a previous community-based cluster trial to promote the mental and physical health of mothers and babies in Victoria reported a range of contextual factors that may have influenced intervention effectiveness, including major local government change at the time of the trial, length of time available for embedding the intervention prior to outcome evaluation and the challenge of changes in role for the MCH nurses themselves. ${ }^{28} 29$

The short time available for embedding the SILC intervention, including a run-in period of just 2 months, may have limited the likelihood of drop-in centres becoming established and known within the community, and also the time for the broader MCHN workforce to be aware of the new service. The 3-year project timeline was determined by the funding body (Department of Education and Early Childhood Development in Victoria).

In terms of blinding, this was not possible at the LGA (randomisation) or cluster levels; however, individual women in the LGAs were not aware of the intervention allocation-the intention was that any trial arm allocation was 'standard' care within the LGA during the intervention period.

\section{CONCLUSION}

Improving breastfeeding rates is a complex and multifaceted endeavour, and in this trial, the complexity was further impacted by the poor compliance with the planned interventions. The additional support we aimed to add to usual care was not provided to the extent 


\begin{tabular}{|c|c|c|c|c|c|c|c|c|}
\hline \multirow[b]{2}{*}{ Factor } & \multicolumn{8}{|c|}{ Any breast milk feeding } \\
\hline & $\mathrm{n}^{\star}$ & $\%$ & OR & $95 \% \mathrm{Cl}$ & p Value & AOR & $95 \% \mathrm{Cl}$ & p Value \\
\hline \multicolumn{9}{|l|}{ Maternal age } \\
\hline$\geq 25$ years & $3678 / 6184$ & 59.5 & Ref & & & & & \\
\hline$<25$ years & $262 / 732$ & 35.8 & 0.38 & 0.32 to 0.45 & $<0.001$ & 0.43 & 0.34 to 0.54 & $<0.001$ \\
\hline \multicolumn{9}{|l|}{ Mother country of birth } \\
\hline Not Australian born & $1439 / 2015$ & 71.4 & ref & & & & & \\
\hline Australian-born & 1995/3899 & 51.2 & 0.42 & 0.37 to 0.48 & $<0.001$ & 0.46 & 0.39 to 0.56 & $<0.001$ \\
\hline \multicolumn{9}{|l|}{ Health Care Card holder } \\
\hline No & $2107 / 3447$ & 61.1 & ref & & & & & \\
\hline Yes & $260 / 619$ & 42.0 & 0.46 & 0.38 to 0.56 & $<0.001$ & 0.58 & 0.48 to 0.71 & $<0.001$ \\
\hline \multicolumn{9}{|l|}{ Father country of birth } \\
\hline Not Australian born & $1171 / 1691$ & 69.3 & ref & & & & & \\
\hline Australian-born & $1738 / 3266$ & 53.2 & 0.51 & 0.41 to 0.62 & $<0.001$ & NS & & \\
\hline \multicolumn{9}{|c|}{ Aboriginal or Torres Strait } \\
\hline \multicolumn{9}{|l|}{ Islander mother } \\
\hline $\begin{array}{l}\text { No } \\
\text { Yes }\end{array}$ & $3258 / 5626$ & 57.9 & ref & & & & & \\
\hline \multicolumn{4}{|l|}{ Gestational age } & 0.36 to 0.80 & 0.003 & & & \\
\hline$\geq 37$ weeks or more & $3688 / 6379$ & 57.8 & ref & & & & & \\
\hline$<37$ weeks & $199 / 434$ & 45.9 & 0.62 & 0.50 to 0.77 & $<0.001$ & 0.73 & 0.54 to 0.97 & 0.03 \\
\hline \multicolumn{9}{|l|}{ Type of birth } \\
\hline Vaginal birth & $2633 / 4475$ & 58.8 & ref & & & & & \\
\hline Caesarean birth & $1258 / 2331$ & 54.0 & 0.82 & 0.75 to 0.90 & $<0.001$ & 0.76 & 0.67 to 0.86 & $<0.001$ \\
\hline \multicolumn{9}{|l|}{ First time mother } \\
\hline No & $1742 / 4145$ & 58.0 & ref & & & & & \\
\hline Yes & $1292 / 2894$ & 55.4 & 0.90 & 0.81 to 0.99 & 0.04 & NS & & \\
\hline
\end{tabular}




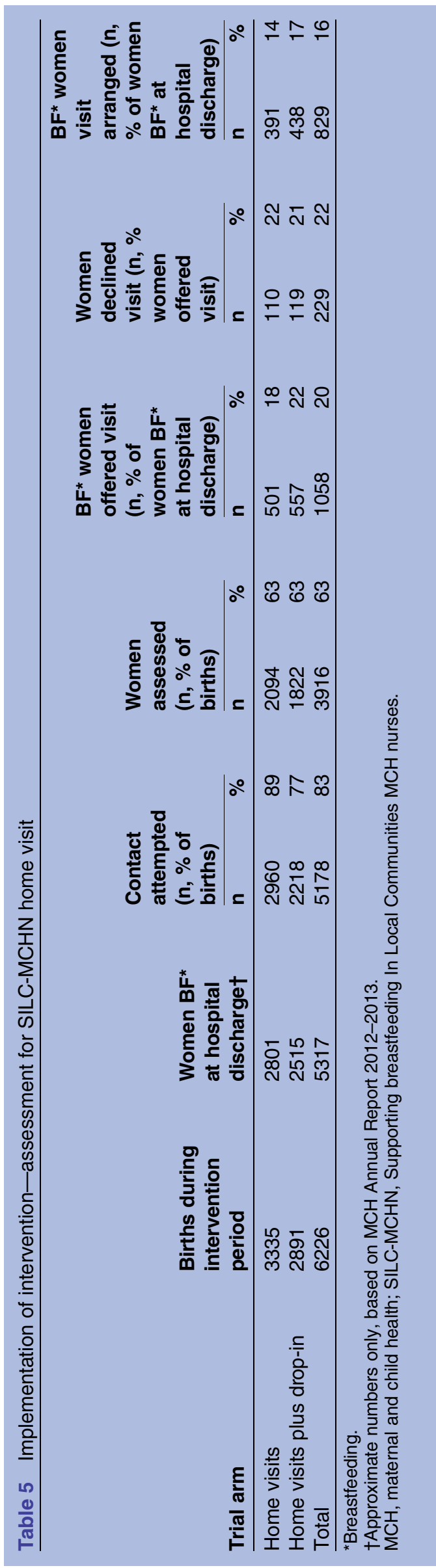

envisaged; only $5 \%$ of women leaving hospital breastfeeding received the intervention as planned, compared with the target of $30 \%$. Within these parameters, in this carefully conducted cluster RCT, extra 'traditional' breastfeeding support, on its own, made no difference to breastfeeding rates as measured in this study. If WHO breastfeeding targets are to be achieved, SILC demonstrates that strategies to improve breastfeeding need to pay careful attention to system-level factors to ensure implementation is normalised and optimal, giving interventions the best chance of succeeding. The importance of social factors in women's decisions about breastfeeding may mean that health professional interventions alone are insufficient to address context-level factors more effectively, so that interventions can be more successfully normalised and given the best chance of being effective.

Acknowledgements The authors gratefully acknowledge the Department of Education and Early Childhood Development, Victoria, Australia for funding SILC. They thank Dr Heather McKay for her contribution to trial co-ordination (maternity leave replacement, July 2012 to March 2013) and Ms Anita Moorhead for her assistance with SILC-MCHN training. Thank you in particular to the women who assisted in piloting the initial documentation; the SILC maternal and child health nurses; the municipality maternal and child health teams and IT staff in all SILC Local Government Areas who facilitated data collection; and the women who responded to the SILC survey. They would also like to thank the members of the SILC Advisory Group and the Municipal Association of Victoria for their input and co-operation; and Xpedite Professional Services Pty Ltd, Data Systems International and Datatime Services Pty Ltd for their services.

Contributors HLM, DAF, LHA and RS conceived and designed the study. HLM, DAF, LHA and RS wrote the initial grant application; LFW was the study statistician who contributed to statistical design and analysis; MC and TS were responsible for day to day management of the trial; LR provided MCH content expertise for intervention implementation; RLC, HLM and MC collected and analysed process evaluation data; HLM, DAF, LHA and RS were responsible for ongoing review of trial processes; HLM, DAF, LHA, RS, MC, RLC and TS developed data collection tools; MC and TS had data collection and management responsibility; DAF, MC, RLC and HLM undertook data analysis. HLM drafted the manuscript and all authors participated in revisions and have approved the final manuscript.

Funding The study was funded by the Department of Education and Early Childhood Development, Victoria, Australia.

Disclaimer The funding body had no role in data analysis or interpretation, nor in manuscript preparation.

Competing interests None declared.

Ethics approval La Trobe University Human Ethics Committee (project number 11-068) and the Department of Education and Early Childhood Development (project number 2011_001305).

Provenance and peer review Not commissioned; externally peer reviewed.

Data sharing statement No additional data are available.

Open Access This is an Open Access article distributed in accordance with the Creative Commons Attribution Non Commercial (CC BY-NC 4.0) license, which permits others to distribute, remix, adapt, build upon this work noncommercially, and license their derivative works on different terms, provided the original work is properly cited and the use is non-commercial. See: http:// creativecommons.org/licenses/by-nc/4.0/

\section{REFERENCES}

1. United Nations Children's Fund (UNICEF). Breastfeeding on the Worldwide Agenda: findings from a landscape analysis on political 
commitment for programmes to protect, promote and support breastfeeding. New York, NY, USA, 2013.

2. National Health and Medical Research Council. Infant Feeding Guidelines: information for health workers. Canberra: National Health and Medical Research Council, 2012.

3. World Health Organization. Expert consultation on the optimal duration of exclusive breastfeeding. Conclusions and recommendations. Secondary expert consultation on the optimal duration of exclusive breastfeeding. Conclusions and recommendations, 2001. http://www. who.int/inf-pr-2001/en/note2001-07.html

4. Australian Institute of Health and Welfare. 2010 Australian National Infant Feeding Survey: indicator results. Canberra: AlHW, 2011.

5. Amir LH, Donath SM. Socioeconomic status and rates of breastfeeding in Australia: evidence from three recent national health surveys. Med J Aust 2008;189:254-6.

6. Amir LH, Forster DA, McLachlan HL, et al. Breastfeeding in Victoria: a report. Secondary breastfeeding in Victoria: a report 2010. http:// www.education.vic.gov.au/healthwellbeing/childyouth/breastfeeding/ default.htm

7. Gagnon AJ, Sandall J. Individual or group antenatal education for childbirth or parenthood, or both. Cochrane Database Syst Rev 2007;(3):CD002869.

8. Lumbiganon $\mathrm{P}$, Martis $\mathrm{R}$, Laopaiboon $\mathrm{M}$, et al. Antenatal breastfeeding education for increasing breastfeeding duration. Cochrane Database Syst Rev 2012;(9):CD006425.

9. Dyson L, McCormick F, Renfrew MJ. Interventions for promoting the initiation of breastfeeding. Cochrane Database Syst Rev 2005;(2): CD001688.

10. Renfrew MJ, McCormick FM, Wade A, et al. Support for healthy breastfeeding mothers with healthy term babies. Cochrane Database Syst Rev 2012;(5):CD001141.

11. Moore ER, Anderson GC, Bergman N, et al. Early skin-to-skin contact for mothers and their healthy newborn infants. Cochrane Database Syst Rev 2012;(5):CD003519.

12. Department of Education and Early Childhood Development. Maternal and Child Health Service. Secondary Maternal and Child Health Service. 2013. http://www.education.vic.gov.au/childhood/ professionals/health/Pages/maternalchildhealth.aspx

13. McLachlan HL, Forster DA, Amir LH, et al. Supporting breastfeeding In Local Communities (SILC): protocol for a cluster randomised controlled trial. BMC Pregnancy Childbirth 2014;14:346.

14. Department of Education and Early Childhood Development (DEECD). Maternal and Child Health Services Annual Report (2009-2010) Secondary Maternal and Child Health Services Annual Report (2009-2010). 2010. http://www.eduweb.vic.gov.au/edulibrary/ public/earlychildhood/mch/report10annualwmr.pdf

15. Consultative Council on Obstetric and Paediatric Mortality and Morbidity 2014, 2010 and 2011, Victoria's Mothers and Babies, Victoria's Maternal, Perinatal, Child and Adolescent Mortality, State Government of Victoria, Melbourne.
16. Forster D, McLachlan H, Lumley J. Factors associated with continuing to feed any breastfeeding at six months postpartum in a group of Australian women. Int Breastfeed $\mathrm{J}$ 2006;1:18.

17. Haggkvist AP, Brantsaeter AL, Grjibovski AM, et al. Prevalence of breast-feeding in the Norwegian Mother and Child Cohort Study and health service-related correlates of cessation of full breast-feeding. Public Health Nutr 2010;13:2076-86.

18. Johns HM, Forster DA, Amir LH, et al. Infant feeding practices in the first 24-48 h of life in healthy term infants. Acta Paediatr 2013;102: e315-20.

19. Schwartz K, D'Arcy HJ, Gillespie B, et al. Factors associated with weaning in the first 3 months postpartum. J Fam Pract 2002;51:439-44.

20. Campbell MK, Grimshaw JM, Elbourne DR. Intracluster correlation coefficients in cluster randomized trials: empirical insights into how should they be reported. BMC Med Res Methodol 2004;4:9.

21. Forster D, McLachlan H, Lumley J, et al. Two mid-pregnancy interventions to increase the initiation and duration of breastfeeding: a randomized controlled trial. Birth 2004;31:176-82.

22. McDonald SJ, Henderson JJ, Faulkner S, et al. Effect of an extended midwifery postnatal support programme on the duration of breastfeeding: a randomised controlled trial. Midwifery 2010;26:88-100.

23. Rossiter JC. The effect of a culture-specific education program to promote breastfeeding among Vietnamese women in Sydney. Int $J$ Nurs Stud 1994;31:369-79.

24. Duffy EP, Percival P, Kershaw E. Positive effects of an antenatal group teaching session on postnatal nipple pain, nipple trauma and breastfeeding rates. Midwifery 1997;13:189-96.

25. Hoddinott $P$, Craig LC, Britten J, et al. A serial qualitative interview study of infant feeding experiences: idealism meets realism. BMJ Open 2012;2:e000504.

26. Campbell M, Fitzpatrick $R$, Haines $A$, et al. Framework for design and evaluation of complex interventions to improve health. BMJ 2000;321:694-6.

27. Hoddinott P, Britten J, Prescott GJ, et al. Effectiveness of policy to provide breastfeeding groups (BIG) for pregnant and breastfeeding mothers in primary care: cluster randomised controlled trial. BMJ 2009;338:a3026.

28. Lumley J, Watson L, Small R, et al. PRISM (Program of Resources, Information and Support for Mothers): a community-randomised trial to reduce depression and improve women's physical health six months after birth [ISRCTN03464021]. BMC Public Health 2006;6:37.

29. Small R, Watson L, Gunn J, et al. Improving population-level maternal health: a hard nut to crack? Long term findings and reflections on a 16-community randomised trial in Australia to improve maternal emotional and physical health after birth [ISRCTN03464021]. PLOS ONE 2014;9:e88457. 